\title{
Medial temporal lobe atrophy in stroke patients: relation to pre-existing dementia
}

\author{
H Hénon, F Pasquier, I Durieu, J P Pruvo, D Leys
}

\begin{abstract}
Objective-The links between stroke and Alzheimer's disease seem to be closer than expected by chance. In a previous study it was shown that up to $16 \%$ of patients admitted for stroke had pre-existing dementia. Medial temporal lobe atrophy (MTLA) is strongly associated with Alzheimer's disease. The aim of this study was to determine the prevalence of MTLA and its relation with pre-existing dementia.

Method-The study was conducted on 170 consecutive stroke patients ( 87 women; median age 75 years; 152 infarcts), who underwent non-contrast CT with temporal lobe oriented $2 \mathrm{~mm}$ contiguous slices at admission. A cut off point of $11.5 \mathrm{~mm}$ was used to differentiate patients with and without MTLA. Pre-existing dementia was assessed using the informant questionnaire on cognitive decline in the elderly (IQCODE) with a cut off score of 104.

Results-Ninety four patients $(55.3 \%)$ had MTLA, of whom $23(24.5 \%)$ had preexisting dementia; of 76 patients without MTLA, only four (5.3\%) had pre-existing dementia $(p=0.0007)$. The logistic regression analysis with MTLA as dependent variable found the following independent variables: increasing age $(p<0.05)$, and global cerebral atrophy scores $(p<0.01)$. The IQCODE scores just reached significance $(\mathrm{p}=0.05)$.
\end{abstract}

Conclusion-Stroke patients with MTLA are more likely to have pre-existing dementia; this suggests that Alzheimer's disease might contribute to the dementia syndrome. A longitudinal follow up is now necessary to determine whether stroke patients with MTLA and without preexisting dementia are at increased risk of Alzheimer's disease over subsequent years. (F Neurol Neurosurg Psychiatry 1998;65:641-647)

Keywords: stroke; dementia; medial temporal lobe atrophy

The prevalence of dementia increases after an ischaemic stroke. ${ }^{1-5}$ Even after exclusion of patients who are demented 3 months after onset of stroke, the relative risk of dementia over a 4 year period of follow up is $5.5 .^{3}$ Many dementias occurring years after an ischaemic stroke have a progressive course and occur without new cerebrovascular events, ${ }^{3}$ suggesting a degenerative rather than a vascular origin. ${ }^{67}$ As Alzheimer's disease is the most frequent cause of degenerative dementia, this finding supports the increasing evidence that the links between stroke and Alzheimer's disease are probably closer than expected by chance. ${ }^{3}$ 7-16 This relation suggests that a high prevalence of Alzheimer's disease in stroke patients is possible. In a recent study, we found that one sixth of consecutive stroke patients aged 40 years or more had pre-existing dementia, ${ }^{6}$ and that pre-existing dementia is associated with increasing age, female sex, global cerebral atrophy, and leukoaraiosis, but not with previous stroke, transient ischaemic attacks, or silent infarcts on CT. ${ }^{6}$ These findings suggest that most pre-existing dementia in stroke patients could be degenerative in origin. Medial temporal lobe atrophy (MTLA) determined by temporal lobe oriented $\mathrm{CT}^{17}$ is strongly associated with Alzheimer's disease in patients attending a memory clinic. ${ }^{18-20}$ It also predicts the development of dementia among those with mild cognitive impairment. ${ }^{21}$ The hypothesis underlying this study is that, if preexisting dementia in stroke patients is associated with Alzheimer's disease pathology, it should also be associated with MTLA. However, to our knowledge, MTLA has never been studied in stroke patients. The responsibility of old strokes in the development of MTLA either by direct involvement or trans-synaptic changes as the relation between leukoaraiosis and MTLA remains questionable.

The aim of this study was to determine in consecutive stroke patients the prevalence of MTLA and the relation with pre-existing dementia.

\section{Methods}

This study was conducted over a 24 week period (1 December 1995-12 May, 1996) in consecutive stroke patients admitted in the acute stroke unit of the Lille University Hospital (France). This unit admits in emergency adult patients, whose onset of pathology is less than 24 hours and likely to be vascular in origin (ischaemic or haemorrhagic). Criteria for stroke were clinical signs of focal disturbance of cerebral function lasting more than 24 hours with no apparent cause other than of vascular origin; criteria for transient ischaemic attacks were episodes of focal cerebral dysfunction, presumably ischaemic in origin, lasting less than 24 hours and followed by total recovery. We excluded from the study patients (1) with transient ischaemic attacks or cerebral venous thrombosis, (2) younger than 40 years, (3) who were not fluent French speakers, (4) who were not white, (5) without a reliable informant, (6) with a history of severe head trauma or cerebral 
neurosurgery, (7) referred from another hospital, to avoid selection bias, and (8) who did not live in the urban community of Lille (1.2 million inhabitants), because the follow up is usually performed in other centres. Previous stroke and transient ischaemic attacks were not exclusion criteria.

Patients were examined at admission by a neurologist in training and a board certified neurologist, and underwent standard blood and urine tests, 12 lead ECG, and noncontrast CT. Doppler ultrasonography and B mode echotomography of the cervical arteries, bidimensional transthoracic echocardiography, and tests for syphilis and hyperfibrinogenaemia were performed within 24 hours. Delayed CT was performed within 8-10 days. Other examinations were performed in selected patients-namely, cerebral MRI, 24 hour ECG, transoesophageal echocardiography, cerebral angiography, and tests for hypercoagulability.

Medical history was determined from all available records (general practitioner's letter or telephone call) and sources (patient, family, or general practitioner). We prospectively collected the following data: age; sex; education level ( $<8$ years of education/ $\geqslant 8$ years of education); first or second degree relative history of migraine, stroke, depression, or dementia; presence of arterial hypertension (defined as systolic blood pressure $\geqslant 160 \mathrm{~mm} \mathrm{Hg}$ or diastolic blood pressure $\geqslant 90 \mathrm{~mm} \mathrm{Hg}$ or current treatment with antihypertensive drugs, either before onset of stroke or lasting more than 1 month after stroke onset); diabetes mellitus (defined as serum glucose concentration $>1.20$ $\mathrm{g} / 1$ or current use of antidiabetic drugs); hyperlipidaemia (defined as fasting serum concentration of triglycerides $>1.5 \mathrm{~g} / 1$ or fasting cholesterol serum concentration $>2.3 \mathrm{~g} / 1$ ); previous transient ischaemic attacks or stroke; mean alcohol consumption $>300 \mathrm{~g} /$ week; cigarette smoking $(>10$ cigarettes a day or cessation $<5$ years earlier), high and low risk cardiopathies according to TOAST criteria ${ }^{22}$; and significant stenosis of the internal carotid arteries defined as a narrowing of $50 \%$ or more of the lumen documented by Doppler ultrasonography, B mode echotomography, magnetic resonance, or conventional angiography. The severity of the clinical deficits was scored according to the rating scale of Orgogozo et al, ${ }^{23}$ which was constructed to include items reflecting global severity and has been shown to have a high interrater reliability for individual items and for the additive score. ${ }^{24}$ The Orgogozo rating scale is a widely distributed and easily applicable scale, largely used in many centres. Data were recorded as soon as possible after onset and always within 24 hours. The presumed causes of stroke were defined at discharge, according to the TOAST criteria. $^{22}$

The assessment of pre-existing dementia was conducted within 48 hours of stroke onset by means of a French translation of the informant questionnaire on cognitive decline in the elderly (IQCODE). ${ }^{25}$ This questionnaire is composed of 26 questions addressed to a close relative about the changes experienced by the patient over the past 10 years, in aspects of daily behaviour requiring memory and other intellectual abilities. Each item is given a score from 1 to 5 ( 1 =has become much better, $2=$ has become a little better, $3=$ has not changed, $4=$ has become a bit worse, $5=$ has become much worse). The global score is the sum of the scores of all items and ranges from 26 to 130 points. This questionnaire has a good interrater and intrarater reliability. ${ }^{26-28}$ There is a good correlation between the score on the mini mental state examination (MMSE) ${ }^{29}$ and IQCODE scores. ${ }^{27} 283031$ This questionnaire is a valuable tool to screen dementia in the community, ${ }^{32}$ and it is not influenced by patients' previous intelligence, level of education, and socioeconomic class ${ }^{26-28} 3031$ because its aim is to detect a decline from a high previous to a lower present intellectual level. ${ }^{25}$ The IQCODE score is as effective as the $\mathrm{MMSE}^{29}$ for the screening of dementia ${ }^{27}$ irrespective of its severity. ${ }^{32}$ The main interest of the IQCODE at the acute stage of stroke, is that it does not require any participation of the patients themselves at the acute stage of the disease when neuropsychological functions are severely influenced by stroke. We classified patients with IQCODE scores of 104 or more as having pre-existing dementia because this cut off score accurately classifies $92.7 \%$ of patients with Alzheimer's disease as actually demented and $88.1 \%$ of the general population sample as normal, leading to a diagnostic accuracy of $90.4 \%{ }^{27}$ The assessment of independent daily living activities was performed with the informant completed measure of activities of daily living and behaviour in elderly patients with cognitive impairment ${ }^{33}$ : the score obtained quantifies the loss of independence in daily living activities (IDLA) with a score varying from $0 \%$ to $100 \%$. This measure quantifies the loss of independent daily living activities and allows a follow up, irrespective of the presence of motor or perceptual handicaps. ${ }^{33}$ A standardised interview of the patient's family was performed to look for depressive symptoms within the past year before stroke. Previous Parkinson's disease was also studied. Tests for vitamin $\mathrm{B}_{12}$ deficiency or thyroid disease were performed in patients with clinical or biological features suggesting such an underlying disease. In patients with pre-existing dementia, these analyses were performed because of the presence of previous cognitive impairment. All patients are followed up every 6 months.

Brain CT was performed without contrast, on an Elscint 2004 Elite Plus machine (Elscint, Israel), by means of $5 \mathrm{~mm}$ contiguous slices. Haemorrhagic infarcts were quoted as infarcts. We determined on CT the number and location of old infarcts defined as infarcts seen at admission on CT and not related to the index stroke. We did not analyse the volume of infarcts because our technique of CT did not allow a post hoc volumetric analysis. The location of old infarcts was determined as a function of the side (right, left) and of the the territory involved (anterior superficial, posterior superficial, deep, cerebellum-brainstem) according to the procedure of Tatemichi et $a l,{ }^{1}$ 
with the addition of small centrum ovale infarcts that we included in the deep infarct group. We defined silent infarcts according to the criteria of Mounier-Vehier et al. ${ }^{34}$ Leukoaraiosis was defined according to the criteria of Inzitari et $a l^{35}$ and scored by means of the 0-3 point rating scale of Blennow et al. ${ }^{36}$ Leukoaraiosis was assessed on the hemisphere opposite to a unilateral focal vascular lesion, if any, and on the right hemisphere in the remainder. Cerebral atrophy was scored according to a global rating method. ${ }^{37}$ This assessment provided cerebral atrophy scores of 0 (no atrophy), 1 (mild atrophy), 2 (moderate atrophy), or 3 (severe atrophy).

We included in the CT procedure at admission investigations of the medial temporal lobe according to the procedure of Jobst et $a l^{18}$; planes were $20^{\circ}$ caudal to the orbitomeatal line in $2 \mathrm{~mm}$ contiguous slices through the posterior fossa. The minimum width of each medial temporal lobe (combined hippocampal formation and parahippocampal gyrus) was measured in $\mathrm{mm}$ at the narrowest point (irrespective of side), level with the brainstem (between the anterior and posterior limits of the brainstem). The whole medial temporal lobe is covered by 5 to 7 scan sections when 2 $\mathrm{mm}$ sections are made, and the minimum thickness is usually found midway through the series. Left and right medial temporal lobe widths (combined hippocampal formation and parahippocampal gyrus) were measured from the film by a neuroradiologist (JPP), blinded to the clinical diagnosis. In another study conducted in our centre with the same procedure, two independent neuroradiologists had an excellent interobserver agreement, with an interrater deviation $<1 \mathrm{~mm}$ and a complete agreement in $92 \%$ of cases. ${ }^{20}$ We used a cut off point of $11.5 \mathrm{~mm}$ to differentiate patients with MTLA (defined as at least one medial temporal width of $\leqslant 11.5 \mathrm{~mm}$ ) from patients without MTLA. The choice of this cut off point has been established according to indications provided by Jobst et al. ${ }^{18}$ We previously found that a cut off point of $11.5 \mathrm{~mm}$ distinguishes patients with Alzheimer's disease from nonAlzheimer's disease patients with a specificity of 0.95 , a sensitivity of 0.81 , a predictive posi-

Table 1 Results of the bivariate analysis comparing demographic characteristics, risk factors for stroke, risk factors for dementia, severity of the neurological deficit at admission, stroke subtype, and cause of stroke between patients with medial temporal lobe atrophy MTLA (+) and patients without (MTLA -)

\begin{tabular}{|c|c|c|c|c|c|}
\hline \multirow{2}{*}{$\frac{\text { Variables }}{\text { Demographic characteristics: }}$} & \multicolumn{2}{|c|}{$M T L A(-) n=76$} & \multirow[t]{2}{*}{$M T L A(+) n=94$} & \multirow[t]{2}{*}{ OR (95 CI) } & \multirow[t]{2}{*}{ p Value } \\
\hline & & & & & \\
\hline Age $(y)^{\star}$ & 68. & $(42-91)$ & $79(49-101)$ & & $<0.0001$ \\
\hline Female sex $\dagger$ & 35 & $(46.1)$ & $52(55.3)$ & $1.5(0.8-2.7)$ & 0.2295 \\
\hline Primary education level† & 63 & $(82.9)$ & $76(80.9)$ & $0.9(0.4-1.9)$ & 0.7315 \\
\hline Loss of IDLA ()$^{\star}$ & 3 & $(0-92)$ & $17(0-85)$ & & $<0.0001$ \\
\hline \multicolumn{6}{|l|}{ Cognitive status before stroke: } \\
\hline IQCODE score* & 78 & $(78-128)$ & $88(78-130)$ & & $<0.0001$ \\
\hline \multicolumn{6}{|l|}{ Possible risk factors for stroke: } \\
\hline Smokers $\dagger$ & 18 & $(23.7)$ & $13(13.8)$ & $0.5(0.2-1.1)$ & 0.0980 \\
\hline Alcoholism $\dagger$ & 21 & $(27.6)$ & $14(14.9)$ & $0.5(0.2-1)$ & 0.0412 \\
\hline Arterial hypertension $\dagger$ & 43 & $(56.6)$ & $52(54.2)$ & $1 \quad(0.5-1.8)$ & 0.8694 \\
\hline Diabetes mellitus $\dagger$ & 6 & $(7.9)$ & $16(17)$ & $2.4(0.9-6.5)$ & 0.0780 \\
\hline Hyperlipidaemiat & 18 & $(23.7)$ & $19(20.2)$ & $0.8(0.4-1.7)$ & 0.5855 \\
\hline Migraineurst & 10 & $(13.2)$ & $11(11.7)$ & $0.9(0.4-2.2)$ & 0.7742 \\
\hline History of atrial fibrillation $\dagger$ & 12 & $(15.8)$ & $25(26.6)$ & $1.9(0.9-4.2)$ & 0.0896 \\
\hline Coronary heart disease $\dagger$ & 18 & $(23.7)$ & $36(38.3)$ & $2(1-3.9)$ & 0.0419 \\
\hline Peripheral artery disease $\dagger$ & 10 & $(13.2)$ & $9(9.6)$ & $0.7(0.3-1.8)$ & 0.4610 \\
\hline Familial stroket & 27 & $(35.5)$ & $31(33)$ & $0.9(0.5-1.7)$ & 0.7276 \\
\hline Familial migraine $\dagger$ & 11 & $(14.5)$ & $11(11.7)$ & $0.8(0.3-1.9)$ & 0.5925 \\
\hline \multicolumn{6}{|l|}{ Possible risk factors for dementia: } \\
\hline History of depression $\dagger$ & 15 & $(19.7)$ & $10(10.6)$ & $0.5(0.2-1.2)$ & 0.0958 \\
\hline Parkinson's disease† & 2 & $(2.6)$ & $2(2.1)$ & $0.8(0.1-5.9)$ & 0.6059 \\
\hline Thyroid disorderst & 6 & $(7.9)$ & $4(4.3)$ & $0.5(0.1-1.9)$ & 0.2491 \\
\hline Familial dementia† & 10 & $(13.2)$ & $13(13.8)$ & $1 \quad(0.4-2.6)$ & 0.8987 \\
\hline Familial depression $\dagger$ & 16 & $(21.1)$ & $20(21.3)$ & $1 \quad(0.5-2.1)$ & 0.4716 \\
\hline Previous stroke or TIA $\dagger$ & 22 & $(28.9)$ & $24(25.5)$ & $0.8(0.4-1.7)$ & 0.6182 \\
\hline \multicolumn{6}{|l|}{ Stroke characteristics: } \\
\hline Orgogozo score at admission ${ }^{\star}$ & 70 & $(10-100)$ & $70(5-100)$ & - & 0.6946 \\
\hline Ischaemic stroke $\dagger$ & 68 & $(89.5)$ & $84(89.4)$ & $1 \quad(0.4-2.7)$ & 0.9812 \\
\hline Cervical artery atheromat & 16 & $(21.1)$ & $21(22.3)$ & $1.1(0.5-2.3)$ & 0.8397 \\
\hline Cardioembolism $†$ & 15 & (19.7) & $27(28.7)$ & $1.6(0.8-3.4)$ & 0.1768 \\
\hline Lipohyalinosis $\dagger$ & 11 & $(14.5)$ & $12(12.8)$ & $0.9(0.4-2.1)$ & 0.7462 \\
\hline Other definite causest & 1 & (1.3) & $1(1.1)$ & $0.8(0.1-13.2)$ & 0.6957 \\
\hline Undetermined cause $†$ & 3 & $(3.9)$ & $7(7.4)$ & $2 \quad(0.5-7.9)$ & 0.2656 \\
\hline Unknown cause & 28 & $(36.8)$ & $24(25.5)$ & $0.6(0.3-1.1)$ & 0.1116 \\
\hline \multicolumn{6}{|l|}{ CT findings: } \\
\hline Leukoaraiosis score ${ }^{\star}$ & 0 & $(0-3)$ & $1(0-3)$ & & 0.0004 \\
\hline Cerebral atrophy score ${ }^{\star}$ & 1 & $(0-3)$ & $2(0-3)$ & & $<0.0001$ \\
\hline At least 1 old infarct $†$ & 28 & $(36.8)$ & $35(37.2)$ & $1.0(0.6-1.8)$ & 0.9580 \\
\hline Anterior superficial infarct + & 1 & $(1.3)$ & $3(3.2)$ & $2.4(0.3-24.0)$ & 0.4224 \\
\hline Posterior superficial infarct $†$ & 3 & (3.9) & $5(5.3)$ & $1.4(0.3-5.9)$ & 0.6745 \\
\hline Superficial infarct $\dagger$ & 4 & $(5.3)$ & $8(8.5)$ & $1.6(0.5-5.6)$ & 0.4111 \\
\hline Deep infarct $\dagger$ & 23 & $(30.3)$ & $26(27.7)$ & $0.9(0.5-1.7)$ & 0.7094 \\
\hline Cerebellum-brainstem $\dagger$ & 4 & $(5.3)$ & $4(4.3)$ & $0.8(0.2-3.4)$ & 0.7577 \\
\hline Right hemispheret & 20 & $(26.3)$ & $28(29.8)$ & $1.1(0.6-2.2)$ & 0.6171 \\
\hline Left hemisphere† & 16 & $(21.1)$ & $20(21.3)$ & $1.0(0.5-2.1)$ & 0.9717 \\
\hline Bilateral infarcts $\dagger$ & 10 & $(13.2)$ & $26(17)$ & $1.3(0.6-3.0)$ & 0.4865 \\
\hline At least 1 silent infarct $\dagger$ & 21 & $(27.6)$ & $34(36.2)$ & $1.3(0.7-2.4)$ & 0.6960 \\
\hline
\end{tabular}

*Median values (range), Mann-Whitney $U$ test.

tNumber of patients (\%); $\chi^{2}$ test with Yates' correction when necessary or Fisher's exact test. - Not applied.

Significant statistical links appear in bold characters. 
tive value of 0.99 , a predictive negative value of 0.45 , and a diagnostic accuracy of 0.83 in patients attending a memory clinic. $^{20}$ In patients with temporal lobe mass effect due to bland or haemorrhagic acute stroke, or with an old infarct involving the medial temporal lobe, the measure was performed only on the unaffected side.

The first step of the statistical analysis consisted of a description of the prevalence of MTLA, with $95 \%$ confidence intervals (95\% CIs). The second step consisted of a bivariate analysis comparing variables between patients with and without MTLA. We used the $\chi^{2}$ test, with Yates' correction or Fisher's exact test when appropriate, and the odds ratio (OR) method with $95 \%$ CIs to compare qualitative factors between groups. We used the MannWhitney $U$ test to compare quantitative variables; variables compared between groups were IQCODE scores, demographic characteristics, risk factors for stroke, risk factors for dementia, severity of the neurological deficit at admission, stroke subtype and cause, and CT data. The third step of statistics consisted of a logistic regression analysis ${ }^{38}$ with MTLA (quoted 1 when present and 0 when absent) as dependent variable. The independent variables included in the multivariate analysis were selected from the bivariate analysis with a 0.25 level as a screening criterion for selection of candidate variables. ${ }^{38}$ Data were analysed with the SPSS/Macintosh package.

\section{Results}

The study population consisted of 170 patients ( 87 women; 83 men) with a median age of 75 years (range 42 to 101 years). Eighteen patients had a deep intracerebral haemorrhage and 152 had an ischaemic stroke. One hundred and twenty four patients had a first ever stroke and 46 had had a previous stroke or transient ischaemic attack. Twenty seven patients had an IQCODE score $\geqslant 104$.

Of 27 patients with prestroke dementia, five had a history of depressive symptoms: however, they did not fulfill the criteria of a major depressive episode and did not require a treatment. We found a history of Parkinson's disease in three of 170 patients, none of them having pre-existing dementia. In the 27 patients with pre-existing dementia, vitamin $\mathrm{B}_{12}$ concentrations and thyroid functions were normal.

Of the 170 patients, 94 (55.3\%; 95\% CI 47.8-62.8) had MTLA. In three patients $(1.8 \%)$, the measure of medial temporal lobe width was performed in only one side because of the existence of oedema involving the temporal lobe on the other side. The breakdown of patients with MTLA in various age categories was 12 of 54 patients between 40 and 69 years $(22.2 \%$; $95 \%$ CI $11.1-3.3), 36$ of 54 patients between 70 and 79 years $(66.7 \%$; 95\% CI: $54.1-79.2$ ), and 46 of 62 patients aged 80 years or more $(74.2 \% ; 95 \%$ CI $63.3-$ 85.1). Of 94 patients with MTLA, 23 (24.5\%; 95\% CI 15.8-33.2) had pre-existing dementia; of 76 patients without MTLA, only $4(5.3 \%$; $95 \%$ CI 0.2-10.3) had pre-existing dementia
Table 2 Results of the logistic regression analysis with MTLA as dependent variable.

\begin{tabular}{lrll}
\hline & \multicolumn{1}{l}{$\beta$} & \multicolumn{1}{l}{$S E$} & $p$ Value \\
\hline Age & 0.0426 & 0.0193 & 0.03 \\
Cerebral atrophy score & 0.6714 & 0.2517 & 0.01 \\
IQCODE score & 0.0317 & 0.0169 & 0.05 \\
Constant & -6.7478 & 1.7359 & 0.0001 \\
\hline
\end{tabular}

$(p=0.0007)$. Two of these four patients had previous stroke or transient ischaemic attack, one had silent infarct on CT. The median value of the IQCODE scores was 88 (range 78-130) in patients with MTLA and 78 (range 78-128) in patients without MTLA $(p<0.0001)$.

The other results of the bivariate analysis are provided in table 1 . The results of the logistic regression analysis with MTLA as dependent variable are given in table 2 . The overall prediction of the mode is $71.2 \%$ with total $\chi^{2}=46.618, d f=5(p=0.0001)$. IQCODE scores just reached significance $(p=0.05)$.

\section{Discussion}

This study showed that MTLA was present in $55.3 \%$ of consecutive stroke patients of 40 years of age or more; patients with MTLA were more likely to have pre-existing dementia; factors independently associated with MTLA were age and global cerebral atrophy, whereas pre-existing cognitive decline just reached significance.

The cut off value of $11.5 \mathrm{~mm}$ provides a good diagnostic accuracy for a diagnosis of Alzheimer's disease in patients attending a memory clinic. ${ }^{20}$ The method is very simple. It yielded a diagnostic accuracy close to those of more complex techniques. ${ }^{16-41}$ Magnetic resonance volumetry is probably more sensitive. However, in stroke patients, MRI is not always feasible at the acute stage of stroke because of contraindications such as pacemakers, availability of the machines in emergency, and poor cooperation in some patients. We could have assessed MTLA by means of delayed MRI but this procedure would have introduced two sources of bias: (1) exclusion of patients with early death and of patients who could not undergo MRI because of poor cooperation and (2) delayed structural changes in the medial temporal lobes in patients with infarcts in the posterior cerebral artery territory. Therefore, for the purpose of this study, CT was the most appropriate procedure.

MTLA was present in $55.3 \%$ of consecutive stroke patients of 40 years of age or more. Our study was performed using CT to allow consecutive recruitment of patients and the French law does not allow the use of radiography in healthy controls. To our knowledge, there are no data available on the prevalence of MTLA in healthy controls using Jobst's method. ${ }^{18}{ }^{20}$ However, MTLA was studied in anxious patients with memory complaints but normal performance on memory tests and in controls free of cognitive complaints who required CT for otological reasons: only $5 \%$ of these patients had MTLA. ${ }^{20}$ Data are available using MRI. Whereas most patients with probable Alzheimer's disease have 
medial temporal lobe atrophy, a significant number of non-demented subjects do as well. ${ }^{42}{ }^{43}$ Launer et $a l^{42}$ found MTLA in $24 \%$ of non-demented controls aged 65 to 85 years. Golomb et $a l,{ }^{43}$ who studied hippocampal atrophy in a sample of healthy volunteers (aged 55 to 88 years), found a prevalence of $33 \%$, increasing with age and reaching $56 \%$ in those 76 years and older. Despite methodological discrepancies (CT $v$ MRI, age of the population), the prevalence of MTLA seems to be higher in our study. The most likely explanation could be high prevalence of patients with Alzheimer's disease in our population.

The brain, medial temporal lobe structures included, atrophies with advancing age in cognitively normal people. Using MRI, Convit et $a l^{44}$ found an age related reduction in medial and lateral lobe volumes. However, age is also a significant risk factor for Alzheimer's disease. Moreover, in healthy people, the volume of the temporal lobe remains constant with aging, whereas there was a significant age related decrease (about $1 \%$ each decade) of posterior frontal lobe volume. ${ }^{45}$ Double $e t a l^{46}$ considered that significant brain atrophy is not a consequence of advancing age and suggest a regional specificity of damage in Alzheimer's disease. In our study, we found pre-existing dementia to be at the limit of significance, independently of the effect of age and cerebral atrophy scores.

Dementia was diagnosed according to the IQCODE, an informant questionnaire filled with a patient's close relative as soon as possible after admission, to evaluate the evolution of cognitive functions over a 10 year period before stroke. ${ }^{25}$ It was not possible to perform a reliable neuropsychological examination of the patients themselves to evaluate their preexisting cognitive status, because of the potential consequences of the stroke lesions. The information of the relatives has proved to be useful for the diagnosis of dementia when recorded in the same unstructured manner as the clinical history, ${ }^{47}{ }^{48}$ as well as in structured interviews used for epidemiological studies, ${ }^{49} 50$ even after death. ${ }^{50}$ This method is as sensitive as the $\mathrm{MMSE}^{29}{ }^{51}$ in screening for mild dementia. ${ }^{32}$ To avoid an overestimation of the prevalence of pre-existing dementia, we used a cut off point of 104 for the IQCODE as used by Jorm and Jacomb, ${ }^{27}$ although the best cut off point to discriminate between demented and non-demented patients with the French version of the IQCODE seems to be $94 .^{52}$ Therefore, according to previous studies, we probably correctly classified $90 \%$ of patients and the risk of misclassification of patients who had an IQCODE score of 104 or more was about $7 \% .{ }^{27}$ Moreover, patients with a high IQCODE score had a more severe impairment in every day functioning, supporting the diagnosis of dementia in patients with high IQCODE score. Our study has probably underestimated the number of patients with dementia before stroke.

In a memory clinic, MTLA is strongly associated with a diagnosis of Alzheimer's disease. ${ }^{20}$ Of 94 stroke patients with MTLA, 23 (24.5\%) had pre-existing dementia, suggesting a contri- bution of Alzheimer pathology to the dementia syndrome; these patients are currently being followed up to determine whether they will fulfill clinical criteria for possible or probable Alzheimer's disease. ${ }^{53}$ Of 76 patients without MTLA, four (5.3\%) had pre-existing dementia. We did not get enough information concerning the course of the disease at the time of the study to be sure of the aetiological diagnosis in these patients. The hypothesis is that they may have vascular dementia, or degenerative dementia other than Alzheimer's disease; two of them had a history of stroke or transient ischaemic attacks, and silent infarcts were present in one. The follow up of the patients is now necessary to confirm this hypothesis. Seventy one patients with MTLA did not meet the criteria for pre-existing dementia; the first possibility is that the IQCODE is not as sensitive or specific for Alzheimer's disease in this group of stroke patients as in a group of patients with memory complaints: MTLA could be a feature of cerebrovascular disease as well as Alzheimer's disease. Using MRI, Laakso et $a l^{4}$ found hippocampal atrophy in seven of nine patients with vascular dementia. However, they used the DSM III R criteria $^{55}$ to diagnose vascular dementia. As these criteria do not require any temporal relation between stroke and cognitive impairment, vascular dementia may have been overdiagnosed and the cases with temporal atrophy might correspond to Alzheimer's disease associated with stroke, as suggested by the authors themselves. Moreover, in another study, ${ }^{56}$ atrophy of the temporal sulci on MRI distinguished patients with Alzheimer's disease from patients with vascular dementia, and no association was found between risk factors for stroke and temporal atrophy. ${ }^{57}$ Very few data are available concerning the relation between white matter abnormalities and MTLA: in a study conducted in healthy elderly volunteers, MTLA on MRI was associated with impaired memory performance whereas periventricular and deep white matter lesions were not ${ }^{58}$; in the study of Launer et $a l,{ }^{42}$ no significant relation was found between MTLA and deep or periventricular white matter lesions seen on MRI. In our study, there was a significant relation between leukoaraiosis and MTLA in univariate analysis; however in the multivariate analysis, when the other variables were taken into account, leukoaraiosis did not appear as a factor independently associated with MTLA. We might hypothesise that some stroke lesions may have induced secondary MTLA. However, this hypothesis is unlikely because (1) CT was performed at admission, before secondary MTLA could occur; (2) in patients with old infarcts affecting the internal temporal lobe, the measure of the width of the medial temporal lobe was performed only on the unaffected side; (3) there was no difference $(p=0.76)$ in the prevalence of MTLA between patients with and without old infarcts on CT. This is in accordance with the data from the literature. In a study conducted in demented and non-demented people, ${ }^{42}$ MTLA was found to be associated with severity of dementia and cortical and white matter 
atrophy, but not with the presence of infarction. The second possibility is that the IQCODE is not as sensitive as a battery of neuropsychological tests to assess the diagnosis of dementia. With a cut off point of 104 for the IQCODE scores we probably underestimated the prevalence of pre-existing dementia in stroke patients. In more recent studies with the IQCODE, a lower cut off point was chosen ${ }^{52}{ }^{59}$; therefore, some patients with MTLA who were classified as non-demented could be misclassified. The last possibility is that patients with MTLA and no pre-existing dementia may be at a preclinical stage of Alzheimer's disease. A longitudinal study has shown that $50 \%$ to $70 \%$ of patients with mild cognitive impairment and MTLA develop Alzheimer's disease after 3 years and $91 \%$ after 4 years of follow up. ${ }^{60} \mathrm{~A}$ recent prospective study using $\mathrm{MRI}^{61}$ showed that loss of temporal volume is an excellent predictor of dementia of the Alzheimer type and may mark the beginning of the disease process within 6 years before the onset of dementia. This could also explain why although patients with MTLA were more likely to be demented before stroke, the IQCODE score just reached significance. The patients at a preclinical stage of Alzheimer's disease who have low IQCODE scores may have skewed the results of the logistic regression analysis towards a weakened link between MTLA and IQCODE scores. Our patients are currently being followed up to determine whether they are at increased risk of Alzheimer's disease in the next few years. These patients might be at increased risk of progressive dementia because of the potential summation of vascular and Alzheimer lesions of the brain. ${ }^{7}$ They may contribute to an important part of the 5.5-fold increased risk of new onset dementia found after an ischaemic stroke. ${ }^{3}$

We did not find any significant relation between the presence of old infarcts on CT and MTLA. We did not study the relation between the MTLA and the size and location of the acute stroke for three reasons: (1) they could not have influenced the pre-existing cognitive status, or the size of medial temporal lobe; (2) it was not possible to assess the location and size of the acute lesions because CT was performed too early after stroke onset when most infarcts are not visible; (3) because of early death, a second CT or MRI could not be performed in all patients.

The classification of the dementia syndromes would be of interest. However, the aetiological diagnosis of the dementia syndrome cannot be easily made at the acute stage of stroke because the neuropsychological evaluation of the patient is much more influenced by the acute effects of stroke than by a potential underlying disease. Our patients are currently being followed up to determine the aetiology of the dementia syndrome (for instance, Alzheimer's disease, frontotemporal dementia, dementia with Lewy bodies, vascular dementia, other dementia) according to the neuropsychological profile, the MRI data, the time course of the disease, and postmortem when possible, and to confirm our hypothesis that degenerative processes might explain many dementias associated with stroke.

In conclusion, the association of MTLA and pre-existing dementia in stroke patients suggests that Alzheimer's pathology contributes to the dementia syndrome in these patients. Whether stroke patients without pre-existing dementia and with MTLA are at increased risk of Alzheimer's disease over the next few years and contribute to the increased risk of new onset dementia in stroke patients remains unknown. A longitudinal follow up is now necessary to determine whether they fulfill criteria for Alzheimer's disease and to obtain neuropathological confirmation when possible.

We thank Drs H Petit and A Destée for their contribution, Drs Godefroy and Duhamel for statistical assistance, and Mrs J Campos for her technical assistance. This study was supported by the direction de la recherche et des études doctorales by the direction de la recherche et des études doctorales
(DRED, grant ER 153), the CH\&U de Lille (grant 9306), the (DRED, grant ER 153), the CH\&U de Lille (grant 9306), the
association pour la recherche et l'enseignement en pathologie association pour la recherche et l'enseignement en pathologie
neurovasculaire (APREPAN,) and the association d'étude et de neurovasculaire (APREPAN, and the association
recherche sur la maladie d'Alzheimer (ADERMA).

1 Tatemichi TK, Foulkes MA, Mohr JP, et al. Dementia in stroke survivors in the stroke data bank cohort. Prevalence, incidence, risk factors, and computed tomographic findings. Stroke 1990;21:858-66.

2 Tatemichi TK, Desmond DW, Mayeux R, et al. Dementia after stroke: baseline frequency, risks, and clinical features in a hospitalized cohort. Neurology 1992;42:1185-93.

3 Tatemichi TK, Paik M, Bagiella E, et al. Risk of dementia after stroke in a hospitalized cohort: results of a longitudinal study. Neurology 1994;44:1885-91.

4 Loeb C, Gandolfo C, Croce R, et al. Dementia associated with lacunar infarction. Stroke 1992;23:1225-9.

5 Kokmen E, Whisnant JP, O'Fallon WM, et al. Dementia after ischemic stroke: a population-based study in Rochester, Minnesota (1960-1984). Neurology 1996;46:154-9.

6 Hénon H, Durieu I, Lucas C, et al. Prevalence of pre-existing dementia in consecutive stroke patients. Neurology 1996;47:852-3.

7 Pasquier F, Leys D. Why are stroke patients prone to develop dementia ? $\mathcal{f}$ Neurol 1997;244:135-42.

8 Tatemichi TK. How acute brain failure becomes chronic: a view of the mechanisms of dementia related to stroke. Neurology 1990;40:1652-9.

9 Jellinger K, Danielczyk W, Fischer P, et al. Clinicopathological analysis of dementia disorders in the elderly. $\mathcal{F}$ Neurol Sci 1990;95:239-58.

10 Erkinjuntti T, Hachinski VC. Rethinking vascular dementia. Cerebrovasc Dis 1993;3:3-23.

11 Saunders AM, Roses AD. Apolipoprotein E allele frequency, ischemic cerebrovascular disease, and Alzheimer's disease [letter]. Stroke 1993;24:1416.

12 Ince PG, McArthur FK, Bjertness E, et al. Neuropathological diagnoses in elderly patients in Oslo: Alzheimer's disease, Lewy body disease, vascular lesions. Dementia disease, Lewy
1995;6:162-8.

13 Victoroff J, Mack WJ, Lyness SC, et al. Multicenter clinicopathological correlation in dementia. Am $\mathcal{F}$ Psychiatry 1995;152:1476-84.

14 Frisoni G, Geroldi C, Blanquetti A, et al. Apolipoprotein e4 allele frequency in vascular dementia and Alzheimer's disease [letter]. Stroke 1994;25:1703

15 Terry JG, Howard G, Mercuri M, et al. Apolipoprotein E polymorphism is associated with segment-specific extracranial carotid artery intima-media thickening. Stroke 1996; A27;1755-9.

16 Frisoni GB, Bianchetti A, Geroldi C, et al. Measures of medial temporal lobe atrophy in Alzheimer's disease. $\mathcal{f}$ Neurol Neurosurg Psychiatry 1994;57:1438-9

17 de Leon MJ, George AE, Stylopoulos LA, et al. Early marker for Alzheimer disease: the atrophic hippocampus. Lancet 1989;ii:672-3.

18 Jobst KA, Smith AD, Szatmari M, et al. Detection in life of confirmed Alzheimer's disease using a simple measurement of medial temporal lobe atrophy by computed tomography. Lancet 1992;340:1179-83

19 Pasquier F, Bail L, Lebert F, et al. Determination of medial temporal lobe atrophy in early Alzheimer's disease with computed tomography. Lancet 1994;343:861-2.

20 Pasquier F, Hamon M, Lebert F, et al. Medial temporal lobe atrophy in memory disorders. $\mathcal{F}$ Neurol 1997;244:175-81.

21 George AE, de Leon MJ, Stylopoulos LA, et al. CT diagnostic features of Alzheimer disease: importance of the choroidal/hippocampal fissure complex. Am 7 Neuroradiol 1990;11:101-7.

22 Adams HP Jr, Bendixen BH, Kappelle LJ, et al. Classification of subtypes of acute ischemic stroke. Definitions for use in a multicenter clinical trial. Stroke 1993;24:35-41.

23 Orgogozo JM, Calpideo R, Anagnosto CN, et al. Mise au point d'un score neurologique pour l'évaluation clinique des infarctus sylviens. Presse Med 1983;12:3039-44. 
24 Orgogozo JM, Dartigues JF. Methodology of clinical trials in acute trials in acute cerebral ischemia: survival, functional and neurological outcome measures. Cerebrovasc Dis 1991, 1(suppl 1):100-11

25 Jorm AF, Korten AE. Assessment of cognitive decline in the elderly by informant interview. Br f Psychiatry 1988;152: 209-13.

26 Jorm AF, Broe GA, Creasey H, et al. Further data on the validity of the informant questionnaire on cognitive decline in the elderly (IQCODE). International fournal of Geriatric Psychiatry 1996;11:131-9.

27 Jorm AF, Jacomb PA. The informant questionnaire on cognitive decline in the elderly (IQCODE): sociodemographic correlates, reliability, validity, and some norms. Psychol Med 1989;19:1015-22.

28 Jorm AF, Scott R, Cullen JS, et al. Performance of the informant questionnaire on cognitive decline in the elderly (IQCODE) as a sreening test for dementia. Psychol Med 1991;21:785-90.

29 Folstein MF, Folstein SE, McHugh PR. Mini mental state: a practical method for grading the cognitive state of
patients for the clinician. FPsychiatr Res 1975;12:189-98.

30 Bowers J, Jorm AF, Henderson S, et al. General practitionner's detection of depression and dementia in elderly patients. Med F A A ust 1990;153:192-6.

31 Mulligan R, Mackinnon A, Jorm AF, et al. A comparison of alternative methods of screening for demntia in clinical settings. Arch Neurol 1996;23:532-6.

32 Morales JM, Gonzalez-Montalvo JI, Bermejo F, et al. The screening of mild dementia with a shortened version on the informant questionnaire on cognitive decline in the elderly. Alzheimer Dis Assoc Disord 1995;9:105-11.

33 Weintraub S. The record of independent living. An informant-completed measure of activities of daily living and behavior in elderly patients with cognitive impairment. American Fournal of Alzheimer's Care and Related Disorders; 1:35-9.

34 Mounier-Vehier F, Leys D, Rondepierre Ph, et al. Silent infarcts in patients with ischemic stroke are related to age and arcts in patients with ischemic stroke are related
size of the left atrium. Stroke 1993;24:1347-51.

35 Inzitari D, Giordano GP, Ancona AL, et al. Leuko-araiosis, intracerebral hemorrhage and arterial hypertension. Stroke intracerebral hem

36 Blennow K, Wallin A, Uhlemann C, et al. White-matter lesions on CT in Alzheimer patients. Relationship to clinical symptomatology and vascular factors. Acta Neuro Scand 1991;83:187-93.

37 Leys D, Pruvo JP, Petit H, et al. Maladie d'Alzheimer: analyse statistique des résultats du scanner X. Rev Neurol (Paris) 1989;145:134-9.

38 Bendel RB, Afifi AA. Comparison of stopping rules in forward regression fournal of the American Statistical Association $1977 ; 72 ; 46-53$.

39 Killiany RJ, Moss MB, Albert MS, et al. Temporal lobe regions on magnetic resonance imaging identify patients with early Alzheimer's disease. Arch Neurol 1993;50:94954 .

40 Scheltens Ph, Leys D, Barkhof F, et al. Atrophy of the medial temporal lobe on magnetic resonance imaging in probable Alzheimer's disease and normal aging: diagnostic value and neuropsychological co

41 Willmer J, Carruthers A, Guzman DA, et al. The usefulness of CT scanning in diagnosing dementia of the Alzheimer type. Can F Neurol Sci 1993;20:210-6.

42 Launer LJ, Scheltens P, Lindeboom J, et al. Medial temporal lobe atrophy in an open population of very old persons: cognitive, brain atrophy, and sociomedical correlates. Neurology 1995; 45:747-62.

43 Golomb J, Leon MJ de, Kruger A, et al. Hippocampal atrophy in normal aging: an association with recent memory impairment. Arch Neurol 1993;50:967-73.
44 Convit A, de Leon MJ, Hoptman MJ, et al. Age-related changes in brain: I. Magnetic resonance imaging measures of temporal lobe volumes in normal subjects. Psychiatr $Q$ 1995;66:343-55.

45 De Carli C, Murphy DGM, Gillette JA, et al. Lack of age-related differences in temporal lobe volume of very healthy adults. Am f Neuroradiol 1994;15:689-96.

46 Double KL, Halliday GM, Kril JJ, et al. Topography of brain atrophy during normal aging and Alzheimer's disease. Neurobiol Aging 1996;17:513-21.

47 Morris JC, Fulling K. Early Alzheimer's disease. Diagnostic considerations. Arch Neurol 1988;45:345-9.

48 Huppert FA, Tym E. Clinical and neuropsychological assessment of dementia. Br Med Bull 1986;42:11-18.

49 Copeland JRMC, Kelleher MJ, Kellet JM. A semistructured clinical interview for the assessment of diagnosis and mental state in the elderly. The geriatric mental state schedule. I. Development and reliability. Psychol Med 1976; 6:439-49.

50 Davis PB, White $\mathrm{H}$, Price JL, et al. Retrospective post-mortem dementia assessment: validation of a new clinical interview to assist neuropathologic study. Arch Neurol 1991;48:613-17.

51 Jorm AF. Methods of screening for dementia: a metaanalysis of studies comparing an informant questionnaire with a brief cognitive test. Alzheimer Dis Assoc Disord 1997; 11:158-62.

52 Law S, Wolfson C. Validation of a French version of an informant-based questionnaire as a screening test for Alzheimer's disease. Br f Psychiatry 1995;167:541-4.

53 McKhann G, Drachman D, Folstein M, et al. Clinical diagnosis of Alzheimer's disease: report of the NINCDSADRDA work group under the auspices of Department of Health and Human Services Task Force on Alzheimer's disease. Neurology 1984;34:939-44.

54 Laakso MP, Partanen K, Riekkinen P, et al. Hippocampal volumes in Alzheimer's disease, Parkinson's disease with and without dementia, and in vascular dementia. An MRI study. Neurology 1996;46:678-81.

55 American Psychiatric Association Committee on Nomenclature and Statistics. Diagnosis and statistical manual of mental disorders (DSM-III-R). 3rd ed, revised. Washington, DC: American Psychiatric Association, 1987.

56 Charletta D, Gorelick PB, Dolleau TJ, et al. CT and MRI findings among African-Americans with Alzheimer's disease, vascular dementia and stroke without dementia. Neurology 1995;45:1456-61.

57 Greewald BS, Kramer-Ginsberg E, Bogerts B, et al. Qualitative magnetic resonance imaging findings in geriatric depression. Possible link between late-onset depression and Alzheimer's disease. Psychol Med 1997;27:421-31.

58 O'Brien JT, Desmond P, Ames D, et al. Magnetic resonance imaging correlates of memory impairment in the healthy elderly: association with medial temporal lobe atrophy but not white matter lesions. International fournal of Geriatric Psychiatry 1997;12:369-74.

59 Del Ser T, Morales JM, Barquero MS, et al. Application of a Spanish version of the informant questionnaire on cognitive decline in the elderly in the clinical assessment of dementia. Alzheimer Dis Assoc Disord 1997;11:3-8.

60 de Leon MJ, Golomb J, George AE, et al. The radiologic prediction of Alzheimer's disease: the atrophic hippocampal formation. Am F Neuroradiol 1993;14:897-906.

61 Kaye JA, Swihart T, Howieson D, et al. Volume loss of the hippocampus and temporal lobe in healthy elderly persons destinated to develop dementia. Neurology 1997;48:1297304. 